\title{
AUS DER PRAXIS
}

\section{Bildungszeitkonten: Betriebliche Verbreitung und Beispiele}

\section{DIE IDEE}

Weiterbildung braucht auf der Nachfrageseite die Ressourcen Zeit, Geld und Motivation, auf der Angebotsseite Organisation, Qualität und Anerkennung. Zur Stärkung der Ressource Zeit ist verschiedentlich der Vorschlag gemacht worden, die auf betrieblichen Arbeitszeitkonten angesammelten Zeitansprüche für betriebliche oder persönliche Weiterbildung zu nutzen (Bildungs- oder Lernzeitkonten) oder sogar alle Freistellungs- und Zeitansprüche betrieblicher, tariflicher oder gesetzlicher Art durch eine zentrale Behörde verwalten und zuteilen zu lassen. ${ }^{1}$ Dadurch könnten die Betriebe die in auftragsstarken Phasen angesammelten Mehrarbeitsstunden in auftragsarmen Zeiten nutzen, um Weiterbildung zu organisieren. ${ }^{2}$

Hinsichtlich der Verwendung von Zeitansprüchen gibt es zwei zentrale Fragen:

(1) Wer hat die Verfügung über die angesammelten Ansprüche: Kann der einzelne Arbeitnehmer nach eigenen Bildungswünschen zugreifen oder werden die Bildungsansprüche vom Arbeitgeber zugeteilt?

(2) Für welche Bildungszwecke können die gesammelten Ansprüche verwendet werden: Für betrieblich notwendige Weiterbildung oder für Weiterbildung der Beschäftigten nach eigenen Bildungswünschen?

Es geht also um die Freizügigkeit der Konten und die Konvertierbarkeit der auf ihnen angesammelten Ansprüche für verschiedene Bildungszwecke.

Für die Nutzung von auf Arbeitszeitkonten angesparter Zeit für eigene Weiterbildung sind keine speziellen Bildungskonten nötig. Voraussetzung sind jedoch betriebliche Regelungen, bei denen die Zeitanteile vom Konto passend für Lage und Dauer der jeweiligen Weiterbildungswün- sche zusammengestellt werden können (stunden-, bzw. tageweise oder zu längeren Zeiträumen geblockt). Spezifische betriebliche Bildungszeitkonten sind dann erforderlich, wenn eine Kofinanzierung der selbst gewählten Weiterbildung durch den Betrieb erfolgt oder wenn - umgekehrt die Beschäftigten sich mit eigener Zeit an der Finanzierung betrieblicher Weiterbildung beteiligen. In diesen Fällen haben Betriebe wie auch Beschäftigte ein spezifisches Interesse an Regelung und Kontrolle von Zeit (und Geld) für Weiterbildung.

\section{VERBREITUNG VON BILDUNGSZEITKONTEN}

Untersuchungen über die Verbreitung von Arbeits- und Bildungszeitkonten in Betrieben ergaben Anfang der 2000er Jahre ein widersprüchliches Bild. Die letzten verfügbaren Daten liegen aus dem Mikrozensus 2004 und einer Auswertung der bei der Bundesagentur für Arbeit (BA) im Jahr 2005 angebotenen Stellen vor: Danach fallen $40 \%$ der männlichen und $35 \%$ der weiblichen Beschäftigten unter Regelungen von Arbeitszeitkonten (der verschiedensten Art $)^{3}$ und es gab in 2,5\% der Betriebe mit knapp $5 \%$ der Beschäftigten Lernzeitkonten, in welcher Form und Praxis auch immer. ${ }^{4}$ Auch die Empirie betrieblicher Fallstudien zeigt eine sehr geringe Verbreitung von Lernzeitkonten: Laut Institut der deutschen Wirtschaft (IW) und des WSI in der Hans-Böckler-Stiftung gab es unter zehn bzw. elf ausgewählten Betrieben mit Arbeitszeitkonten jeweils nur einen Betrieb, in dem diese Konten zumindest zeitweise für Weiterbildung genutzt worden sind. ${ }^{5}$

\section{BETRIEBLICHE VEREINBARUNGEN}

Dieses Bild wird durch die im Archiv betrieblicher Vereinbarungen in der Mitbestimmungsförderung der Hans-Böckler-
Stiftung vorliegenden Betriebsvereinbarungen zur Weiterbildung (1999 und 2004) und zu Arbeitszeitkonten (2005) bestätigt. Sie stammen aus Akquisen des Archivs bei jeweils mehr als 20.000 Betriebs- und Personalräten in Betrieben mit über 150 Beschäftigten. Unser Archiv von Vereinbarungen erhebt keinen Anspruch auf Vollständigkeit oder statistische Repräsentativität. Angesichts der hohen Anzahl an Betrieben, die bei den regelmäßigen Ak-

1 Vgl. Senatsverwaltung für Arbeit, Soziales und Frauen (2001): Lernen, ein Leben lang, Berlin; Expertenkommission Finanzierung Lebenslangen Lernens (2004): Finanzierung Lebenslangen Lernens - der Weg in die Zukunft, Bielefeld; Bundesministerium für Bildung und Wissenschaft (2008): Empfehlungen des Innovationskreises Weiterbildung für eine Strategie zur Gestaltung des Lernens im Lebenslauf, Bonn.

2 Vgl. Seifert, H. (2006): Lernzeitkonten - ein Instrument zur Förderung der betrieblichen Weiterbildung?, in: Bundesinstitut für Berufsbildung (Hrsg.): Kosten, Nutzen, Finanzierung beruflicher Weiterbildung, Bonn, S. 164.

3 Statistisches Bundesamt (2005): Leben und Arbeiten in Deutschland. Ergebnisse des Mikrozensus 2004, Wiesbaden, S. 47.

4 Zickert, K. (2007): Förderung der beruflichen Qualifizierung durch Weiterbildungs- und Arbeitszeitmanagement, in: IAB-Forschungsbericht 11, S. 48 .

5 Institut der deutschen Wirtschaft/WSI in der Hans-Böckler-Stiftung (2003): Investive Arbeitszeitpolitik - Ansätze für eine Verbindung von Arbeitspolitik und beruflicher Weiterbildung. Online-Veröffentlichung, http://www.arbeitszeiten. nrw.de/pdf/Projekt-Abschlussbericht_Investive_ Arbeitszeitpolitik.pdf, S. 95ff. und S. 193

Winfried Heidemann, Dr., Referatsleiter Qualifikation in der Abteilung Mitbestimmungsförderung der Hans-Böckler-Stiftung. Arbeitsschwerpunkte: Analysen und Institutionenberatung zu Lebenslangem Lernen, Beschäftigungsfähigkeit, Beschäftigungssicherung, Soziale Unternehmensverantwortung.

e-mail: Winfried-Heidemann@boeckler.de 
quisen angeschrieben werden, zeichnen diese Vereinbarungen aber ein materialreiches und differenziertes Bild der betrieblichen Entwicklungen, das im Übrigen auch aus unseren Erfahrungen in der Beratung für Betriebsräte bestätigt wird. Die geringe Zahl von Vereinbarungen zu Lernzeitkonten im Bestand unseres Archivs haben wir im Herbst 2008 durch gezielte Recherchen über unsere Netzwerke der Betriebsräteberatung ergänzt.

So liegen uns zur Nutzung von Arbeitszeitkonten für Weiterbildung zwei Gruppen von Vereinbarungen vor:

(1) Vereinbarungen über Langzeitkonten: In diesen Arbeitszeitvereinbarungen ist die Nutzung für Bildungszwecke nur in einigen wenigen Fällen explizit angesprochen: Unter 64 Langzeitvereinbarungen aus den Jahren 1997 bis 2006 gibt es nur zwei mit ausdrücklich erwähnter Nutzung für Weiterbildung. ${ }^{6}$ Seitdem sind im HBS-Archiv drei weitere Vereinbarungen dieses Typs hinzugekommen. Sie stammen aus den Branchen Zeitarbeit, Fahrzeugbau, Verkehrsdienstleistung, Chemische Industrie und Kraftfahrzeug-Handwerk. In den Vereinbarungen werden die Ziele der Konten und die Bedingungen für Ansparen und Entnahme von Zeitansprüchen geregelt. Weiterbildung ist dabei nur einer von mehreren wählbaren Verwendungszwecken, weitere sind Blockfreizeiten und die Inanspruchnahme vorzeitigen Ruhestands.

Vergleichbar mit diesen Vereinbarungen ist ein Fall eines öffentlichen Versorgungsunternehmens mit einer nicht förmlich geregelten Praxis der Nutzung von LangzeitArbeitszeitkonten für Weiterbildung.

\section{(2) Vereinbarungen zu betrieblichen Qualifizierungsprojekten:}

In dieser Gruppe von Vereinbarungen aus fünf Betrieben wird bei der Organisation von Weiterbildung auf anderweitig geregelte Arbeitszeitkonten zurückgegriffen. Unter den 290 Weiterbildungsvereinbarungen, die wir 1999 ausgewertet haben, gab es nur vier, in denen Arbeitszeitkonten für betriebliche Weiterbildung genutzt wurden. Zwei davon waren Unternehmensvereinbarungen der jeweils zuständigen $\mathrm{Ge}$ werkschaft und zwei Vereinbarungen stammen aus dem gleichen Unternehmen. ${ }^{7}$ In einer zweiten Auswertung von 244 nach 1999 abgeschlossenen Vereinbarungen befand sich keine einzige mit dieser Verknüpfung. ${ }^{8}$ Eine Auswertung von 146 betriebli- chen Vereinbarungen, die anlässlich einer WSI-Befragung von Betriebs- und Personalräten 2007 gesammelt worden sind, förderte nur zwei Vereinbarungen zutage, in denen Zeitaufwendungen für Weiterbildung über ein Arbeitszeitkonto abgerechnet werden. ${ }^{9}$

Die Vereinbarungen dieser Gruppe kommen aus den Bereichen Mineralölindustrie, IT-Branche, Unternehmensdienstleistungen, Medizintechnik und Maschinenbau. In ihnen geht es um die Ziele von Weiterbildung, ihre Durchführung und die Modalitäten der Inanspruchnahme von Zugangsmöglichkeiten.

\section{REGELUNGEN IN VEREINBARUNGEN}

Ziele von Arbeitszeitkonten:

Die mit den Arbeitszeitkonten verbundenen Ziele werden in allgemeiner Weise benannt: Abstimmung von betrieblichen Anforderungen und persönlicher Lebensplanung, Verbesserung der Beschäftigungsfähigkeit, Gestaltung des Übergangs in den Ruhestand, allgemeine Förderung der persönlichen Weiterbildung, Deckung erhöhten Weiterbildungsbedarfs.

Bei den Zielen der Weiterbildung unterscheiden die Vereinbarungen zwischen persönlicher und betrieblich notwendiger Qualifizierung. Es geht um die Förderung persönlicher Weiterbildungsbemühungen, die Sicherung betrieblicher Kompetenzbedarfe und von Standort und Beschäftigung. Vorrangig ist in den Vereinbarungen die selbstgewählte Weiterbildung, nur in einem Falle geht es um die Beteiligung der Beschäftigten an den Kosten der betrieblichen Weiterbildung.

\section{Ansparen von Zeitansprüchen:}

Quellen für das Ansparen von Zeitansprüchen auf den Konten sind Überträge aus Kurz- und Gleitzeitkonten, Mehrarbeitsstunden aus Projekt- und Sonderarbeiten und - in begrenztem Umfang - auch Urlaubstage. Eine neuere Vereinbarung bietet die Möglichkeit an, neben Zeiteinheiten auch Geldbestandteile einzubringen, und zwar zusätzliche Einmal- und Sonderzahlungen sowie Teile des Regelentgelts bis zu einer bestimmten Höhe. In dem betreffenden Großunternehmen der Chemischen Industrie werden auch die Zeitansprüche in Geldeinheiten verbucht und auf dem Konto verzinst.

Einen Sonderfall stellt ein Unternehmen des Maschinenbaus dar, in dem gere- gelt wird, dass die Weiterbildung als reguläre Arbeitszeit gilt und dass darüber hinausgehende Zeitanteile positiv auf dem Arbeitszeitkonto verbucht werden.

\section{Entnahme von Zeitrechten:}

Die Bedingungen für die Entnahme von Zeitansprüchen sind zum Teil detailliert geregelt. Die Nutzung für selbst gewählte Weiterbildungsteilnahme ist immer an betriebliche Absprachen oder individuelle Vereinbarungen mit dem Arbeitgeber gebunden. Eine „freie“, bedingungslose Entnahme geben die Betriebsvereinbarungen nicht her. In einem Betrieb wird auch reguläre betriebliche Weiterbildung, die in einem Weiterbildungsgespräch zwischen Vorgesetzten und Beschäftigten vereinbart wird, durch Entnahmezeiten vom Arbeitszeitkonto durch die Beschäftigten mitfinanziert.

In den Vereinbarungen zur Weiterbildung werden für die Entnahme von Zeitanteilen genaue Regelungen der Kostenteilung zwischen Arbeitgeber und Beschäftigten getroffen. Als Faustregel gilt: Je mehr eine Weiterbildung dem individuellen Fortbildungsinteresse dient, umso höher ist der Zeitanteil, der vom Arbeitszeitkonto abgebucht wird.

\section{BEISPIELE}

Drei Beispiele sollen die skizzierten Entwicklungen illustrieren: Eines steht für die Nutzung von Arbeitszeitkonten für persönliche Weiterbildung, ein zweites für eine differenzierte Kostenteilung zwischen Arbeitgeber und Betriebsrat und ein drittes für die Nutzung von Zeitansprüchen für die betriebsnotwendige Weiterbildung.

\section{(1) Nutzung von Arbeitszeitkonten für} persönliche Weiterbildung:

In einem kommunalen Versorgungsunternehmen gibt es eine Praxis der Nutzung vorhandener Arbeitszeitkonten für persön-

\footnotetext{
6 Böker, K.-H. (2007): Flexible Arbeitszeit - Langzeitkonten, Reihe Betriebs- und Dienstvereinbarungen, Frankfurt, S. 18 und 49.

7 Nachauswertung von: Heidemann, W. (2000): Betriebs- und Dienstvereinbarungen Betriebliche Weiterbildung, Düsseldorf

8 Nachauswertung von: Busse, G./Heidemann, W. (2005): Betriebliche Weiterbildung, Reihe Betriebs- und Dienstvereinbarungen, Frankfurt

9 Busse G./Seifert H. (2009): Tarifliche und betriebliche Regelungen zur beruflichen Weiterbildung, Düsseldorf, S. 73.
} 
liche Weiterbildung, die nicht formell durch Vereinbarung geregelt ist. ${ }^{10}$

Das Unternehmen bietet Interessenten für eine länger dauernde Aufstiegsfortbildung (beispielsweise Meisterkurse) im Rahmen eines bestehenden Jahresarbeitszeitkontos die Möglichkeit, einen sechsmonatigen Vollzeitkurs zu absolvieren. Die dafür benötigten rund 1.000 Arbeitsstunden werden durch maximal drei Wochen Jahresurlaub (ggf. aus zwei Jahren) und ein durch Mehrarbeit aufgebautes Arbeitszeitguthaben von bis zu 250 Stunden gespeist. Wenn das Guthaben aufgebraucht ist, geht das Unternehmen mit einer Art „Zeitkredit“ in Vorleistung, indem die Beschäftigten einen negativen Saldo aufbauen können. Während der Dauer der Fortbildung wird das Gehalt weitergezahlt. Im Vorfeld finden zur Klärung der Vorstellungen an die Zeit nach der Fortbildung Gespräche mit den Interessenten über mögliche - und nicht mögliche - Verwendungsoptionen im Betrieb statt.

\section{(2) Kostenteilung zwischen Arbeitgeber} und Betriebsrat:

In einem Unternehmen der Medizintechnik wurden 2004 vor dem Hintergrund eines Unternehmens-Tarifvertrages zur Standortsicherung Vereinbarungen zum Mitarbeitergespräch und über betriebliche Fortbildungsmaßnahmen abgeschlossen. ${ }^{11} \mathrm{Um}$ Weiterbildung, die im Mitarbeitergespräch vereinbart wird, die aber auch im persönlichen Fortbildungsinteresse liegt, wahrnehmen zu können, muss das Arbeitszeitkonto belastet werden. Dabei gelten differenzierte Regeln, die sich an der verbreiteten Praxis der betrieblichen Weiterbildung orientieren: Bei Weiterbildungsbedarfen, die unmittelbar zur Arbeitsaufgabe gehören, gehen die zeitlichen und sächlichen Kosten voll zulasten des Arbeitgebers. Bei Weiterbildung, die auch dem individuellen Fortbildungsinteresse des Beschäftigten dient, übernimmt der Betrieb zwar die Kosten der Maßnahme, die Beschäftigen müssen aber einen Eigenbeitrag in Höhe der Hälfte des Zeitvolumens aus dem Konto der flexiblen Arbeitszeit einbringen. Diese kofinanzierte persönliche Weiterbildung kann maximal 5 Tage pro Jahr betragen, bewegt sich also im Volumen der meisten Weiterbildungsgesetze der Länder.

(3) Nutzung von Zeitansprüchen für die betriebsnotwendige Weiterbildung: In einem Betrieb des Kfz-Handwerks wurde 2007 auf der Grundlage eines Weiterbil- dungstarifvertrags zwischen Innung und zuständiger Gewerkschaft in einer Betriebsvereinbarung die Monatsarbeitszeit um bis zu 2,5 Stunden für Zwecke der Weiterbildung verlängert. Ausdrückliches Ziel ist die Beteiligung der Arbeitnehmer an den Weiterbildungsaufwendungen der Betriebe. Über die Verwendung der angesammelten Zeitguthaben für welche Art von Weiterbildung wird im Rahmen eines jährlichen Personalgesprächs entschieden, auf das die Beschäftigten Anspruch haben.

\section{ERFAHRUNGEN UND AUSBLICK}

Die verbreiteten Arbeitszeitkonten - insbesondere Langzeitkonten - können grundsätzlich für betriebliche oder eigene Weiterbildung der Beschäftigten genutzt werden. Die von uns ausgewerteten Vereinbarungen und die skizzierten Beispiele zeigen Regelungsmöglichkeiten der betrieblichen Akteure auf, wenn sie die Nutzung von Arbeitszeitkonten für Weiterbildung wünschen. Ein weiteres betriebliches Beispiel aus einem Unternehmen mit einer Weiterbildungspraxis von mehr als 20 Jahren macht darüber hinaus deutlich: Angebote, die der persönlichen Weiterentwicklung dienen, wurden durch Einbringen eigener Zeit angenommen, wenn sie hinreichend attraktiv sind. Dadurch wurde in diesem Unternehmen zeitweise durchaus ein Anstoß für die Entwicklung persönlicher Weiterbildung gegeben. ${ }^{12}$

Insgesamt sind jedoch betriebliche Vereinbarungen über die Verwendung von Arbeitszeitkonten ausdrücklich für Weiterbildungszwecke außerordentlich selten. Soweit sie für Bildungszwecke genutzt werden können, zeigt die Praxis, dass dies kaum geschieht. Eine Recherche bei den Akteuren der vorliegenden Vereinbarungen im Herbst 2008 hat ergeben: Die Nutzung von Arbeitszeitkonten für Weiterbildung beschränkt sich bisher auf wenige Einzelfälle. Nennenswerte Erfahrungen mit dem Instrument „Arbeitszeitkonten für Weiterbildung" liegen also nicht vor.

Für die geringe Inanspruchnahme von gegebenen Möglichkeiten werden seitens der Betriebe drei Gründe genannt:

(1) der Grundsatz, dass betrieblich notwendige Weiterbildung sowohl in Zeit als auch in Geld vom Arbeitgeber zu finanzieren ist. Diese Regel wird von den betrieblichen Akteuren hoch gehalten, auch wenn die Praxis inzwischen wesentlich differen- zierter geworden ist. Jedenfalls ist hier die Ursache zu suchen, warum die modellhaft vorgeschlagene Verschiebung von Qualifizierung in konjunkturell schwächere Zeiten unter Nutzung von Zeitansprüchen der Arbeitnehmer ${ }^{13}$ bisher nicht vorzufinden ist und wohl auch in der aktuellen Wirtschaftskrise kaum genutzt werden wird.

(2) In vielen Großbetrieben und in Ländern mit gesetzlichen Freistellungsmöglichkeiten für Weiterbildung gibt es alternative Möglichkeiten der Weiterbildung für persönliche Interessen, sodass man dafür nicht das Arbeitzeitkonto belasten muss.

(3) Wenn in den Vereinbarungen als Alternativen für Weiterbildung die Umwandlung von Zeitansprüchen in Geld oder ihre Nutzung für vorzeitigen Ruhestand stehen, werden diese in aller Regel den Bildungszwecken vorgezogen. ${ }^{14}$ Deshalb hat der Beraterkreis der Hans-Böckler-Stiftung zum Innovationskreis Weiterbildung der Bundesministerin für Bildung und Forschung vorgeschlagen, die Nutzung von Arbeitzeitkonten für Bildungszwecke zu privilegieren: Wenn eine festzulegende Mindestanzahl von Stunden aus Arbeitzeitkonten für Weiterbildung eingesetzt wird, soll es eine finanzielle Förderung der gewählten Bildungsmaßnahme geben. ${ }^{15}$ Dafür könnten öffentliche und betrieblich oder tarifvertraglich vereinbarte Mittel genutzt werden. Eine solche Möglichkeit der Förderung könnte einen Anstoß geben für die verstärkte Verwendung von Arbeitszeitkonten für persönliche Weiterbildung.

10 Vgl. Ullenboom, D. (2008): "Lernzeiten bei der Stadtwerke Dinslaken $\mathrm{GmbH}^{\prime}$. Abrufbar im Internet. URL: http://www.boeckler.de/pdf/mbf_ netzwerke_stadtwerke_dinslaken_2008.pdf:

11 Vgl. Lux, B. (2007): Fraport AG: Dräger Medical AG \& Co.KG: Standort- und Beschäftigungssicherung und Qualifizierung, in: Hans-Böckler-Stiftung (Hrsg.): Arbeitspapier 149, Düsseldorf.

12 Es handelt sich um den bereits häufig zitierten Weiterbildungstarifvertrag der Deutschen Shell aus dem Jahre 1988. Hier können die Beschäftigten die tariflich verkürzte Arbeitszeit nutzen, um an Weiterbildung teilzunehmen.

13 Vgl. Fußnote 2 .

14 Hildebrandt, E. (2007): Geld ist wichtiger als Zeit, in: WZB-Mitteilungen 117, Berlin.

15 Sehrbrock, I./Heidemann, W. (2007): Schwerpunkte für eine Politik des Lebenslangen Lernens. Empfehlungen an den Innovationskreis Weiterbildung des BMBF, Arbeitspapier der Hans-BöcklerStiftung 152, Düsseldorf. 